\title{
Clinical experience of lomitapide therapy in patients with homozygous familial hypercholesterolaemia
}

\author{
Marina Cuchel $^{\mathrm{a}, *}$, Dirk J. Blom ${ }^{\mathrm{b}, \mathrm{c}}$, Maurizio R. Averna ${ }^{\mathrm{d}}$ \\ ${ }^{a}$ Institute for Translational Medicine and Therapeutics, and Department of Medicine, University of Pennsylvania, 8039 Maloney Building, \\ 3600 Spruce Street, Philadelphia, PA 19104, USA \\ ${ }^{\mathrm{b}}$ University of Cape Town, Cape Town, South Africa \\ ${ }^{\mathrm{c}}$ Medical Research Council of South Africa, Cape Heart Group, Cape Town, South Africa \\ ${ }^{\mathrm{d}}$ Università di Palermo, Palermo, Italy
}

\begin{abstract}
The microsomal triglyceride transfer protein (MTP) inhibitor lomitapide is a licenced adjunct to a low-fat diet and other lipid-lowering medication, with or without low-density lipoprotein apheresis, for the treatment of adults with homozygous familial hypercholesterolaemia (HoFH). In a recently published phase 3 study, patients with HoFH received lomitapide in addition to maximally tolerated lipid-lowering therapy. Treatment with lomitapide resulted in a mean approximate 50\% reduction in LDL-C levels after 26 weeks compared with baseline levels $(p<0.0001)$. This decrease in LDL-C was maintained at Weeks 56 and $78(44 \%[p<0.0001]$ and 38\% [ $p=0.0001]$, respectively). This paper offers clinical perspectives based on selected case histories of patients participating in the phase 3 lomitapide study. These cases provide illustrative examples of the efficacy of lomitapide, with or without apheresis, and show that the effective management of adverse effects can enable patients to remain on effective treatment.
\end{abstract}

(C) 2014 Elsevier Ireland Ltd. All rights reserved.

Keywords: Homozygous familial hypercholesterolaemia; Microsomal transfer protein inhibitor; Lomitapide; Liver function tests; Apheresis; Low fat diet; LDL-cholesterol

\section{Introduction}

Homozygous familial hypercholesterolaemia $(\mathrm{HoFH})$ is characterised by markedly elevated low-density lipoprotein cholesterol (LDL-C) levels and increased cardiovascular disease burden. Although currently available lipid-lowering drugs and apheresis have significantly improved the prognosis for patients with $\mathrm{HoFH}$, most patients with the disease still have LDL-C levels far above the target level of $<2.5 \mathrm{mmol} / \mathrm{L}[1,2]$, and will experience progressive atherosclerosis and cardiovascular complications [2]. Furthermore, apheresis is not universally available and is

\footnotetext{
* Corresponding author. Tel.: +1 215746 2834; fax: +1 2156156520 .

E-mail address: mcuchel@mail.med.upenn.edu (M. Cuchel).
}

not suitable for all patients. Thus despite advances in the treatment of HoFH, management of the condition remains problematic, and novel treatment approaches are urgently required.

Pathophysiologically HoFH is characterised by impaired LDL receptor function, with phenotypic variation driven by wide heterogeneity in mutation profiles [3]. In general, patients carrying mutations in the gene encoding for LDL receptor have been historically characterised as either receptor-defective $(<20 \%$ of normal LDL-receptor function) or receptor-negative ( $<2 \%$ of normal LDL-receptor function). As standard lipid lowering drugs, such as statins or ezetimibe, act mainly via mechanisms that lead to up-regulation of the LDL receptor, it is not unexpected that the response to these treatments among patients with $\mathrm{HoFH}$ 

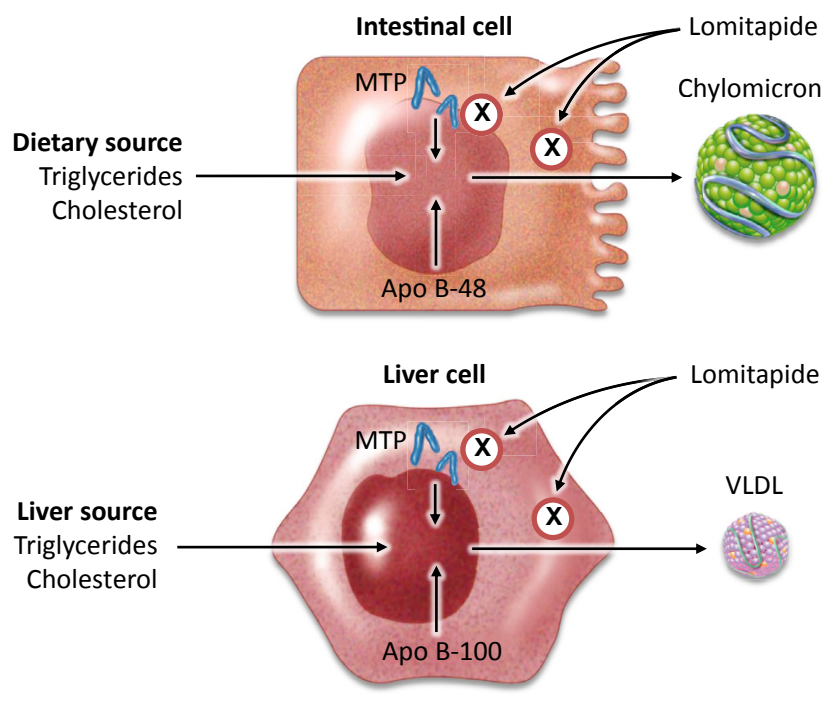

Fig. 1. Lomitapide inhibits the microsomal triglyceride transfer protein (MTP) activity in the liver and in the intestine.

is often poor. Unsurprisingly, $\mathrm{HoFH}$ patients who are LDLreceptor negative are less likely to respond to such agents than patients with defective LDL-receptor function [4]. Similar results are also observed with newer lipid-lowering agents that are currently in development, such as monoclonal antibodies to proprotein convertase subtilisin/kexin 9 (PCSK9) that also act by increasing the number of LDL receptors [5].

Recent research has focussed on investigating other mechanisms of lowering LDL-C levels in HoFH patients that do not rely on functioning LDL receptors, including inhibition of lipoprotein synthesis. Two proteins have been targeted in this effort: 1 . apolipoprotein $\mathrm{B}_{100}\left(\operatorname{apoB}_{100}\right.$; an essential structural component of LDL-C) and its precursor, very low-density lipoprotein (VLDL); and 2. microsomal triglyceride transfer protein (MTP) - a key protein in the assembly and secretion of apoB-containing lipoproteins in the liver and intestine. Mipomersen, an antisense oligonucleotide, was developed to reduce LDL-C by inhibiting the synthesis of apoB ${ }_{100}$ in the liver. In a phase 3 clinical trial in patients with HoFH the mean change in LDL-C from baseline to the end of the 26-week treatment period was $25 \%$ with mipomersen compared with $3.7 \%$ with placebo
[6]. In this paper we describe recent clinical experience with lomitapide, which reduces plasma levels of LDL-C by inhibiting the activity of MTP in the liver and the intestine, thereby inhibiting the synthesis of VLDL and chylomicrons (Fig. 1). Lomitapide has received regulatory approval in the European Union, the United States of America, Canada and Mexico for use under a restricted program as add-on treatment in patients with HoFH.

The overall findings and aggregate data from a singlearm open-label phase 3 study of lomitapide in $\mathrm{HoFH}$ patients are published and will be reviewed only briefly here. More details of the study may be found in the primary publication [7]. This paper offers clinical perspectives based on case studies of individual patients who received lomitapide during the phase 3 study, and illustrates the efficacy of the drug, in patients treated with and without apheresis. The cases also demonstrate how effective management of adverse effects can enable most patients to remain on lomitapide to control their condition.

\section{Overview of phase 3 study [7]}

\subsection{Study design and patient disposition}

Male and female patients aged 18 years or older were eligible for inclusion in the study if they had a diagnosis of $\mathrm{HoFH}$ according to clinical criteria, fibroblast activity or documented mutations in both alleles of the LDL-receptor or alleles known to affect LDL receptor functionality.

Because the study design did not include a placebo treatment arm (Fig. 2), lipid-lowering therapies, including apheresis, had to be stable before lomitapide was initiated. This was achieved by including a minimum 6-week run-in period during which background lipid-lowering therapies were stabilised and patients were established on a low-fat diet (less than 20\% energy from fat per day) to mitigate any gastrointestinal adverse events that might be expected to occur with lomitapide.

The run-in period was followed by an efficacy phase of 6 months duration (Week 26), during which the dose of lomitapide was titrated from $5 \mathrm{mg}$ daily to a maximum of $60 \mathrm{mg}$ daily while concomitant lipid-lowering therapies were kept stable. The aim of the dose titration was to establish an individual 'maximal tolerated dose' for each

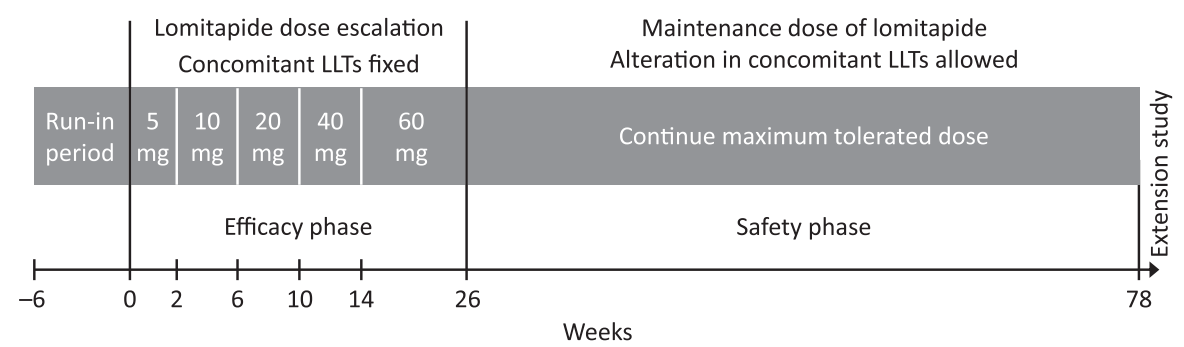

Fig. 2. Design of phase 3 study of lomitapide in patients with homozygous familial hypercholesterolaemia. 
Table 1

Baseline characteristics and treatment history of subjects in phase 3 study of lomitapide $(n=29)$.

\begin{tabular}{lc}
\hline Age (years), mean (range) & $30.7(18-55)$ \\
Male, $n(\%)$ & $16(55 \%)$ \\
History of cardiovascular disease, $n(\%)$ & $27(93 \%)$ \\
- Coronary artery disease & $21(72 \%)$ \\
- Valvular disease & $21(72 \%)$ \\
CABG surgery, $n(\%)$ & $10(34 \%)$ \\
At 21 years of age or younger & $5(17 \%)$ \\
Before 8 years of age & $3(10 \%)$ \\
Multiple CABG surgeries & $3(10 \%)$ \\
Coronary angioplasty, $n(\%)$ & $3(10 \%)$ \\
Aortic valve replacement, $n(\%)$ & $3(10 \%)$ \\
Mitral valve replacement or repair, $n(\%)$ & $3(10 \%)$ \\
Genetic diagnosis, $n(\%)$ & $29(100 \%)$ \\
- LDL R mutations & $28(97 \%)$ \\
True homozygous & $8(29 \%)$ \\
Compound heterozygous & $20(71 \%)$ \\
- LDLRAP1 true homozygous & $1(3 \%)$ \\
Receiving lipid-lowering drugs, $n(\%)$ & $27(93 \%)$ \\
- Statin & $27(93 \%)$ \\
- Ezetimibe (+statin) & $22(76 \%)$ \\
- Bile acid sequestrants & $1(3 \%)$ \\
- Nicotinic acid & $3(10 \%)$ \\
- Fibrates & $1(3 \%)$ \\
Receiving apheresis, $n(\%)$ & $18(62 \%)$ \\
Total cholesterol (mmol/L), mean (range) & $11.1(4.9-18.6)$ \\
LDL-cholesterol (mmol/L), mean (range) & $8.7(3.9-14.6)$ \\
\hline
\end{tabular}

CABG, coronary artery bypass graft; LDL, low-density lipoprotein; ARH, autosomal recessive hypercholesterolaemia.

patient as determined by the LDL-C reduction achieved, an assessment of liver function tests and the occurrence of gastrointestinal symptoms precluding further dose increases. From Week 26 to Week 78 (safety phase) patients continued to receive their maximum tolerated dose of lomitapide and changes in concomitant lipid-lowering therapies were allowed. For eligible patients, there was an open label extension study following the safety phase. Of the 32 persons screened, 29 entered the efficacy phase of the study. Twenty-three patients completed both the efficacy phase and safety phases.

\subsection{Baseline characteristics}

Patient characteristics and treatment history at baseline are summarised in Table 1. Although genetic diagnosis was not a requirement for inclusion, all 29 patients had a confirmed genetic diagnosis of HoFH. Of the 29 patients, 28 (97\%) had mutations in the LDLR gene, and one had a homozygous LDLRAP1 mutation: eight of the patients with LDLR mutations (29\%) were true LDL-receptor homozygotes, $20(71 \%)$ were compound heterozygotes. The majority of patients $(27 ; 93 \%)$ had cardiovascular disease: 21 patients (72\%) had coronary artery and/or valvular disease. Twenty-seven patients (93\%) were receiving lipid lowering therapies at baseline: all of these were receiving a statin; $22(76 \%)$ were also receiving ezetimibe; three (10.3\%) were also receiving nicotinic acid in addition to ezetimibe; one (3\%) was receiving a bile acid sequestrant (colesevelam); and one (3\%) was receiving a fibrate. Eighteen patients $(62 \%)$ were receiving apheresis [8].

The mean baseline LDL-C concentration was $8.7 \mathrm{mmol} /$ L. However, the observed levels varied widely between patients ranging from approximately $3.9 \mathrm{mmol} / \mathrm{L}$ to over $14.2 \mathrm{mmol} / \mathrm{L}$ (Fig. 3). These findings are comparable with published studies that have reported LDL-C concentrations of between $5.6 \mathrm{mmol} / \mathrm{L}$ and $15.5 \mathrm{mmol} / \mathrm{L}$ in treated $\mathrm{HoFH}$ patients $[2,5,9,10]$.

\subsection{Efficacy of lomitapide}

The primary efficacy endpoint of the phase 3 study was the change in LDL-C at the end of the 26-week efficacy phase compared with baseline. Mean decrease in LDL-C compared with baseline was 50\% $(p<0.0001)$ [7]. The

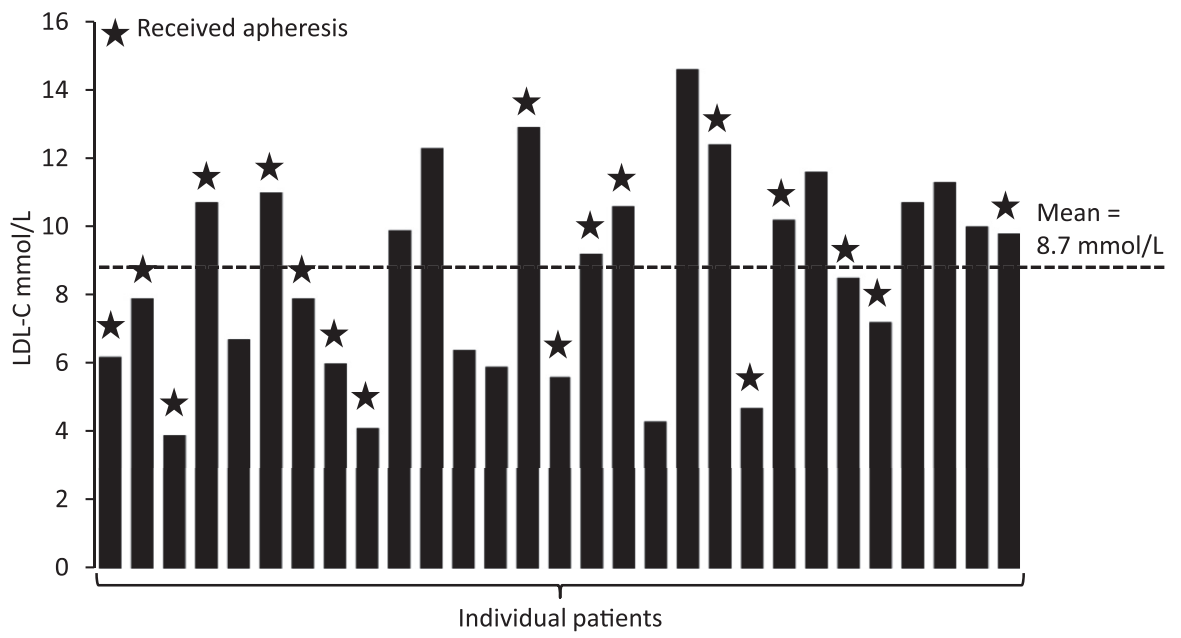

Fig. 3. Low-density lipoprotein (LDL) cholesterol levels of patients at baseline. 

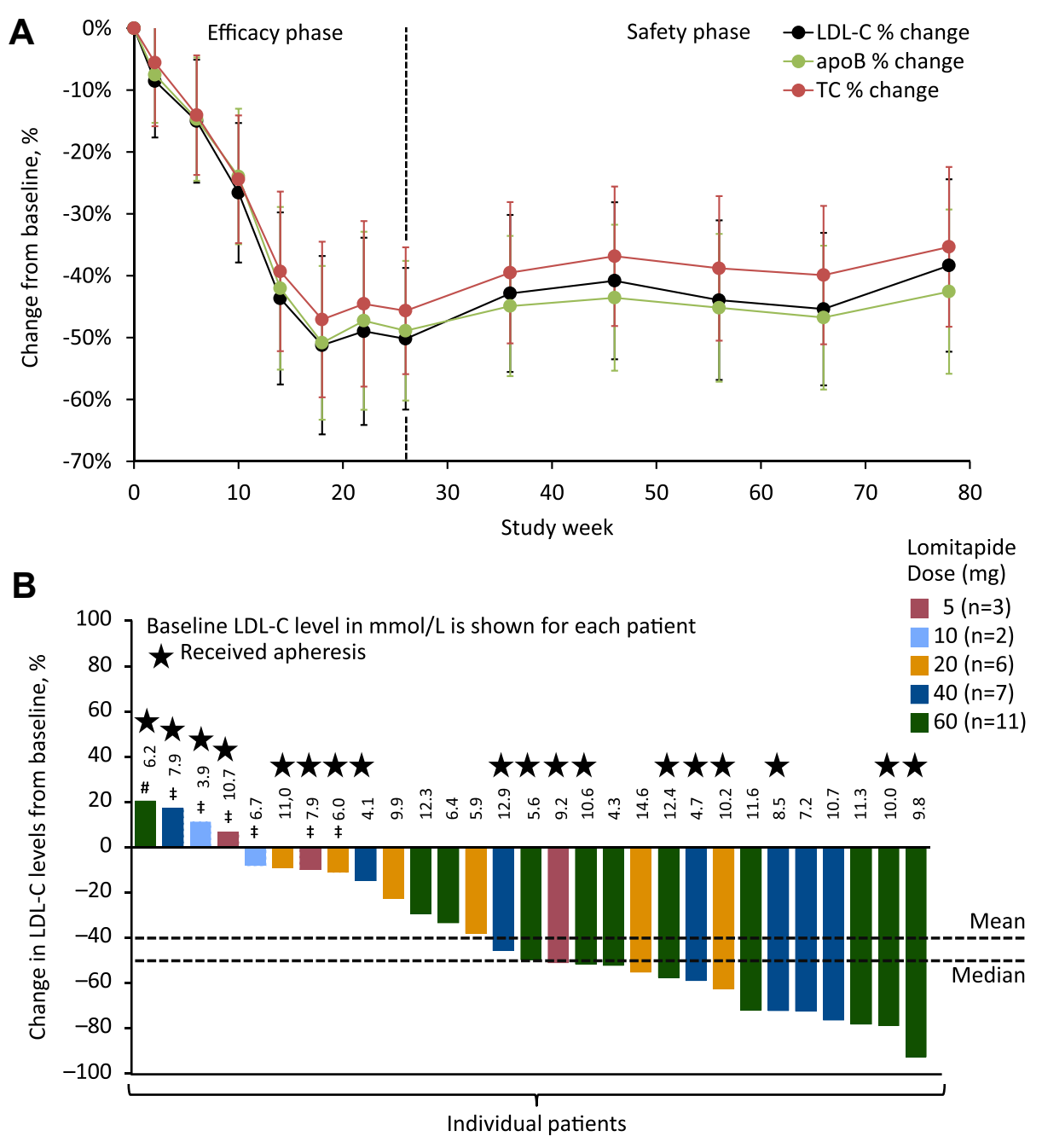

Fig. 4. Percentage reduction in concentration of low-density lipoprotein cholesterol (LDL-C), apolipoprotein B (apoB) and total cholesterol (TC) levels with lomitapide in addition to maximum lipid-lowering therapy (A); individual patient LDL-C responses by dose of lomitapide (B). A. Data are mean, 95\%CI $(n=23)$. B. \#Patient was a responder at later time points during the study. $\ddagger$ Patients discontinued from the study. Numbers over the bars are baseline LDL-C in $\mathrm{mmol} / \mathrm{L}$.

corresponding values after 56 and 78 weeks were $44 \%$ $(p<0.0001)$ and $38 \%(p=0.0001)$, respectively. Fig. 4A shows the percentage reduction in concentration of LDL-C, apolipoprotein B (apoB) and total cholesterol levels with lomitapide in addition to maximum lipid lowering therapy in the 23 patients who completed 78 weeks of treatment and Fig. 4B shows individual patient LDL-C responses at the maximally tolerated lomitapide dose.

The European Society of Cardiology/European Atherosclerosis Society guidelines for the management of dyslipidaemias recommend target LDL-C levels of $<1.8 \mathrm{mmol} /$ $\mathrm{L}(\sim 70 \mathrm{mg} / \mathrm{dL})$ for patients with very high cardiovascular risk and $<2.5 \mathrm{mmol} / \mathrm{L}(\sim 100 \mathrm{mg} / \mathrm{dL})$ for patients with high cardiovascular risk [1]. Table 2 shows the number of patients who achieved these LDL-C targets at different time points during the phase 3 study. Between Weeks 0 and 26 of the study (efficacy phase), 15 patients had achieved the target of $<2.5 \mathrm{mmol} / \mathrm{L}$ and eight achieved the target of $<1.8 \mathrm{mmol} / \mathrm{L}$, and between Weeks 0 and 78 (efficacy and safety phases) the corresponding values showed a slight increase to 16 and nine patients, respectively.

The effects of lomitapide on LDL-C and other lipid parameters were independent of apheresis treatment, whereby Week 26 LDL-C levels were reduced by $48 \%$ in patients not undergoing apheresis versus $55.1 \%$ in those receiving the treatment $(p=0.545)$ [8]. At enrolment, 18 of the 29 patients were receiving either plasma or LDL apheresis. Five of these withdrew from the study during the efficacy phase, while 13 entered the safety phase and completed the study. During the safety phase, when concomitant lipid-lowering therapy could be modified, at the discretion of their physicians and based on the LDL-C levels obtained, three patients discontinued apheresis and a further three reduced the frequency of apheresis while maintaining a good response. 
Table 2

Number of patients achieving European Society of Cardiology/European Atherosclerosis Society target concentrations for low-density lipoprotein cholesterol (LDL-C) [1] during the phase 3 study.

\begin{tabular}{lll}
\hline & $\begin{array}{l}\mathrm{LDL}-\mathrm{C}<2.6 \mathrm{mmol} / \mathrm{L} \\
(<100 \mathrm{mg} / \mathrm{dL})\end{array}$ & $\begin{array}{l}\mathrm{LDL}-\mathrm{C}<1.8 \mathrm{mmol} / \mathrm{L} \\
(<70 \mathrm{mg} / \mathrm{dL})\end{array}$ \\
\hline $\begin{array}{l}\text { Patients achieving } \\
\text { target at any } \\
\text { time point }\end{array}$ & 15 & 8 \\
$\quad \begin{array}{l}\text { Weeks 0-26 } \\
\text { Total number of } \\
\text { patients achieving } \\
\text { target at any } \\
\text { time point } \\
\text { Weeks 0-78 }\end{array}$ & 16 & 9 \\
\hline
\end{tabular}

Case study 1 demonstrates the efficacy of lomitapide in a female patient with HoFH and long-standing cardiovascular disease. Lipid-lowering efficacy was observed both in presence and absence of apheresis treatment.

Case study 1: Lipid response can be maintained with reduced treatment burden

This case describes a 44-year old woman diagnosed with $\mathrm{HoFH}$ at 29 years of age. She is a true homozygote for the S156L LDL-receptor mutation. She had extensive cardiovascular disease having undergone the first of three coronary artery bypass grafts at the age of 27 years in addition to aortic and mitral valve replacements.

Lipid-lowering therapy at baseline comprised rosuvastatin $40 \mathrm{mg}$ daily, ezetimibe $10 \mathrm{mg}$ daily, colesevelam $3750 \mathrm{mg}$ daily and LDL apheresis every 4 weeks. Although this apheresis regimen is considered suboptimal, adverse effects meant that this was the most intensive the patient could tolerate (she developed severe nausea, chest pain and abdominal pain during apheresis that limited her compliance with the treatment). In addition to lipid-lowering therapies, the patient was also taking warfarin, aspirin, metoprolol and ferrous sulphate.

Despite receiving maximum lipid-lowering therapy, the patient's lipids at baseline were still far from target: LDL-C $11 \mathrm{mmol} / \mathrm{L}$, total cholesterol $13 \mathrm{mmol} / \mathrm{L}$, triglycerides $1.9 \mathrm{mmol} /$ $\mathrm{L}$, HDL-C $1.4 \mathrm{mmol} / \mathrm{L}$ (taken just before apheresis). At Week 22, after receiving lomitapide $60 \mathrm{mg}$ for 8 weeks, her LDL-C was $1.1 \mathrm{mmol} / \mathrm{L}$ (Fig. 5). Given this result and her poor tolerance to LDL apheresis, in agreement with her physician and the sponsor, this was her last apheresis treatment. Additionally, based on her results at Week 26 (LDL-C $0.72 \mathrm{mmol} / \mathrm{L})$, it was decided to discontinue treatment with colesevelam from Week 31 onwards.

\section{Concluding comments}

Lomitapide is markedly efficacious in reducing LDL-C both in the presence or absence of apheresis treatment. The phase 3 study showed that some patients can reach LDL-C levels below the goals established by the European Society of Cardiology/European Atherosclerosis Society guidelines [11]. The burden of $\mathrm{HoFH}$ and its treatment on the patient should not be underestimated [12] and treatment changes such as those described in this case may have a significant positive impact on a patient's quality of life, while maintaining acceptable LDL-C levels.

\subsection{Safety and tolerability of lomitapide}

During the phase 3 study, the most frequently reported treatment-emergent adverse events were gastrointestinal, including diarrhoea, nausea, abdominal discomfort, and vomiting. Gastrointestinal adverse events were generally mild to moderate in intensity and were usually managed by dietary review and reinforcement of a low fat diet and occasionally by temporary dose reduction or interruption. Gastrointestinal adverse events were the most frequent reasons for failure to escalate the lomitapide dose to $60 \mathrm{mg}$

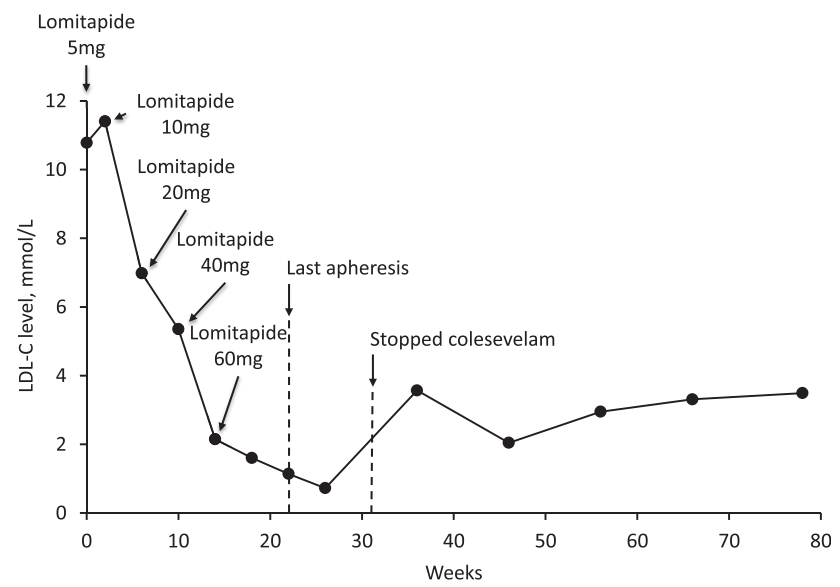

Fig. 5. Case study 1: Low-density lipoprotein cholesterol (LDL-C) level in a 44 year old woman with homozygous familial hypercholesterolaemia receiving lomitapide in phase 3 study. The dose of lomitapide was uptitrated to $60 \mathrm{mg}$ during the efficacy phase of the study (Weeks 0-26). 
(the mean dose taken by patients at the end of the efficacy phase was $38.4 \mathrm{mg}$ in the ITT population and $44.6 \mathrm{mg}$ in the completer population) and contributed to early discontinuation of lomitapide treatment in four patients during the 26-week efficacy phase [7]. The frequency and intensity of gastrointestinal adverse events tended to decrease with time and they did not result in any further discontinuations during the safety phase. These observations suggest that over time patients may have learned to manage their diet better and were increasingly adhering to a low-fat diet to avoid gastrointestinal adverse events. Additionally, gastrointestinal adaptation may have contributed to the improved tolerability.

Side effects can impair a patient's adherence to lomitapide treatment and limit the maximum tolerated dose, potentially reducing efficacy. Case study 2 illustrates how effective management of gastrointestinal adverse effects enabled a patient with receptor-negative $\mathrm{HoFH}$ and no access to apheresis to remain on a dose of lomitapide that reduced his LDL-C by more than $50 \%$.

\section{Case study 2: Careful management of gastro- intestinal adverse events can increase adher- ence and improve treatment response}

This case describes a 27-year old man of Indian ancestry who is a compound heterozygote (mutation $\mathrm{M}-21 \mathrm{~L}$ and mutation $\mathrm{C} 371 \mathrm{X}$ ), resulting in receptor-negative $\mathrm{HoFH}$. The patient was treated with rosuvastatin $40 \mathrm{mg}$ daily and ezetimibe $10 \mathrm{mg}$ daily and did not have access to apheresis. His baseline lipid profile on pharmacotherapy was: LDL-C $=12.8 \mathrm{mmol} / \mathrm{L}$, total cholesterol $14.01 \mathrm{mmol} / \mathrm{L}$, triglycerides $0.77 \mathrm{mmol} / \mathrm{L}$ and $\mathrm{HDL}-\mathrm{C}=0.90 \mathrm{mmol} / \mathrm{L}$.

There was a delay of about 2-3 months before a good response to lomitapide was seen due to initial poor adherence to the treatment (Fig. 6A). However, in the long-term the patient showed a sustained reduction in LDL-C of around 58\% (Fig. 6B) and also significant regression of cutaneous xanthomata.

The initial adherence issues were due to gastrointestinal adverse events. Lomitapide inhibits MTP in the liver and in the intestine and high fat meals can provoke bloating, diarrhoea, and other gastrointestinal symptoms. As an integral part of the lomitapide phase 3 study, patients received dietetic education and counselling about the need to restrict dietary triglyceride intake to $<20 \%$ energy from fat. For this patient, dietary advice was specifically adapted for the Indian style of cooking, including use of ghee (problematic due to high fat content) and spices (not problematic and can be used freely).

Gastrointestinal adverse events, including bloating, nausea and diarrhoeal episodes, occurred on up-titration of lomitapide from $20 \mathrm{mg}$ to $40 \mathrm{mg}$. They were managed with more intensive dietary counselling, occasional use of loperamide to manage the diarrhoea and, (on one occasion) interruption of drug treatment for a few days, followed by downtitration to $20 \mathrm{mg}$ and then up to $40 \mathrm{mg}$ again.

The patient's adherence improved with time, as dietary education resulted in fewer side effects. However, the dose was not increased beyond $40 \mathrm{mg}$ per day, as the patient indicated that he felt unable to take a higher dose.

\section{Concluding comments}

Patients should be advised that gastrointestinal side effects are likely to occur but can be managed by following a low fat diet supplying $<20 \%$ energy from fat per day [13]. In addition, clinical trial data suggests gastrointestinal side effects may improve over time. Knowledgeable dietary input from a dietician is invaluable and there should be a plan for the management of side effects. Both patient and doctor commitment to therapy is essential.

Liver function tests were monitored carefully during treatment with lomitapide during the phase 3 study. Four patients experienced transient alanine transaminase (ALT) elevation of $\geq 5 \times$ ULN. All were managed successfully with dose reduction according to a specific dosing algorithm, which required lomitapide dose reduction for ALT or aspartate transaminase (AST) elevations from $>5 \times$ ULN to $\leq 10 \times$ ULN and treatment interruption/discontinuation for ALT/AST $>10 \times$ ULN $[7,8]$. If levels returned to $<5 \times$ ULN patient could be rechallenged with lomitapide. Bilirubin did not change significantly during lomitapide treatment and no patients discontinued lomitapide treatment based on liver function test elevations. Based on the mechanism of action of lomitapide, hepatic fat accumulation is an expected adverse event. Hepatic fat content was $1 \%$ at baseline and increased to a mean of $8 \%$ at Week 26, but appeared to stabilise thereafter.

Case study 3 reports the successful management of a transaminitis flare with subsequent rechallenge with lomitapide. This case also highlights the importance of 
being vigilant in monitoring potential drug-drug interactions.

\section{Case study 3: Management of transaminase elevation}

This case describes a 55-year old man with $\mathrm{HoFH}$, a compound heterozygote with one South African founder mutation FH Afrikaner 1 (D206E) and a Dutch mutation, FH Amsterdam (S285L). The patient had ischaemic heart disease and had undergone a coronary artery bypass graft procedure. He also had a mild mixed depression/anxiety condition. During the run-in period, the patient had a mild elevation in transaminase levels (Fig. 7) but the levels were within protocol limits. He received lomitapide $5 \mathrm{mg}$ QD for 3 days before drug was interrupted due to an increased ALT of $5.8 \times$ ULN and AST of $2.3 \times$ ULN based on a sample taken at the baseline visit prior to initiation of lomitapide. The event of increase transaminases was assessed as non-serious, and unrelated to lomitapide - most likely caused when his psychiatrist switched his antidepressant medication to fluvoxamine. The patient was monitored and the ALT level decreased, however, in consultation with the study hepatologist of the Drug Safety Monitoring Board, it was decided to perform a liver biopsy to investigate the liver architecture before exposing the patient to lomitapide. The biopsy showed minimal steatosis, no excessive fibrosis and no other significant abnormalities. Lomitapide was subsequently initiated. On treatment with lomitapide the patient showed a profound response in LDL-C, which decreased to approximately $2 \mathrm{mmol} / \mathrm{L}$ while receiving lomitapide $40 \mathrm{mg}$ (the dose was maintained thereafter). During the safety phase there were some spikes in transaminases up to $3-5 \times$ ULN but the patient remained well and therapy continued unchanged. At a routine visit, at which the patient was asymptomatic, and after 2.5 years on lomitapide treatment, the transaminases were noted to have increased to approximately $20 \times$ ULN (ALT reached $954 \mathrm{U} / \mathrm{L}$ ). Fig. 8 shows the patient's transaminase levels during the titration phase (A), the maintenance phase (B) and during the transaminitis flare $(\mathrm{C})$.
The patient reported that his psychiatrist had recently switched his antidepressant medication to agomelatine. As the patient was due to leave for an overseas holiday neither he nor the psychiatrist discussed this change with the study site. Agomelatine has been reported to cause ALT elevation, especially in patients with a background of steatosis (Howland, 2011). Following the patient's return from his vacation he was prescribed clarithromycin (a potent inhibitor of the cytochrome P450 3A4 liver enzyme) by his general practitioner for an upper respiratory tract infection. Lomitapide is metabolised via the cytochrome P450 3A4 liver enzyme and concomitant administration with cytochrome P450 3A4 inhibitors should be avoided, as mandated by the product label $[13,14]$. The combination of these drug treatments, along with lomitapide had most likely caused the ALT flare. Lomitapide, agomelatine and clarithromycin were all stopped and transaminase levels decreased rapidly.

Following return of transaminases to baseline levels the patient asked if he could resume lomitapide treatment. A second liver biopsy was done to evaluate any interval change in hepatic architecture. The liver biopsy showed a slight increase in steatosis but little inflammatory activity or fibrosis. After discussion with the patient the benefits of lomitapide therapy were thought to outweigh the hepatic risk and the patient was rechallenged with lomitapide. The lomitapide dose was titrated back up to $40 \mathrm{mg}$. No further ALT/AST spikes were seen up to the time he died of sudden cardiac death (judged unrelated) at Week 174 (Fig. 9).

\section{Concluding comments}

Transaminase elevations are relatively common and generally dose related. They can be managed by dose reduction/interruption and patients can usually be re-challenged successfully. Specifically, in clinical practice prescription information recommends dose reduction in presence of AST/ALT elevation $>3 \times$ ULN and the dose interruption if levels exceed $5 \times$ ULN $[13,15]$. In the case described here, the transaminitis flare, in which ALT was elevated to $>20 \times$ ULN, appears to have been caused by a concomitant administration of clarithromycin and agomelatine with lomitapide, highlighting the importance of watching for potential drug-drug interactions. 
Finally, Case study 4 illustrates important communication points to inform discussions that clinicians may have with their patients about lomitapide treatment.

\section{Case study 4: Communication points con- cerning lomitapide therapy}

A 23-year old man, a compound heterozygote for two mutations of LDLR gene (FH Palermo-3an exon 13-15 deletion; and FH Palermo 1 a G528D Gly-Asp substitution), had a clinical history of severe hypercholesterolaemia from the age of 7 years and coronary and extracoronary atherosclerosis. The patient smoked 20 cigarettes per day, weighed $58 \mathrm{~kg}$ and had a BMI of 21.9. He was normotensive (blood pressure $120 / 70 \mathrm{mmHg}$ ) and had no concomitant diseases. At baseline he was receiving combination therapy with simvastatin $40 \mathrm{mg}$ and ezetimibe $10 \mathrm{mg}$ daily and weekly LDL apheresis. His LDL-C levels at baseline were $22 \mathrm{mmol} / \mathrm{L}$.

Communication point 1: "Why should I start lomitapide therapy when I am already receiving LDL-apheresis?"

European Society of Cardiology/European Atherosclerosis Society Guideline recommendations for LDL-C clinical targets in patients with dyslipidaemias [1] are: treatment is aimed at reaching LDL-C goals for high-risk subjects of $<2.6 \mathrm{mmol} / \mathrm{L}$, or in the presence of cardiovascular disease or very high-risk subjects $<1.8 \mathrm{mmol} / \mathrm{L}$. If these targets cannot be reached, maximal reduction in LDL-C should be considered using appropriate drug combinations in tolerated doses.

Communication point 2: "Now that I have reached never-seen-before LDL-C levels, should I stop apheresis?"

In a recent publication [16] the targets for apheresis were defined as:

(i) an acute reduction in total cholesterol of $\geq 65 \%$ or LDL-C $\geq 70 \%$ on average during each procedure;

(ii) an interval mean total cholesterol of $<7 \mathrm{mmol} / \mathrm{L}$ or $\mathrm{LDL}-\mathrm{C}<6.5 \mathrm{mmol} / \mathrm{L}$ (or decreases of $>60 \%$ or $>65 \%$, respectively from baseline values off all treatment);

(iii) a baseline level of total cholesterol of $<9 \mathrm{mmol} / \mathrm{L}$ or LDL-C $<8.5 \mathrm{mmol} / \mathrm{L}$ (or decreases of $>50 \%$ or $>55 \%$, respectively from baseline values off all treatment).
The authors acknowledge that in patients receiving apheresis "the levels proposed will probably only postpone rather than prevent cardiovascular disease in most instances but advances in adjuvant drug therapy such as microsomal triglyceride transfer protein and anti-sense $a p o B$ oligonucleotides may enable more radical reductions in $L D L$ to be achieved in the future." [16].

Apheresis is first line treatment for HoFH; however, for this patient a decision was made to stop apheresis treatment when the patient's pre-apheresis LDL-C reached $2.28 \mathrm{mmol} / \mathrm{L}$ (Week 34 of study). This decision was taken after consideration of the rebound kinetics of LDL-C. In this case, using the formula set out by Kroon et al., [16,17] it was estimated that the time averaged value of LDL-C could not reach target levels even if apheresis was intensified to twice weekly, and it was therefore probable that apheresis was making no meaningful contribution to the patient's treatment. The patient was advised that apheresis remains a viable treatment option depending on his clinical follow-up. Fig. 10 summarises the patient's mean LDL-C levels during treatment with lomitapide, showing that the lipid response was maintained even after discontinuation of apheresis. These findings are in accordance with the results of a post-hoc analysis of the phase 3 study data, which showed no effect of apheresis on efficacy, in terms of reduction in LDL-C [8].

\subsection{Conclusions of phase 3 study of lomitapide}

The addition of lomitapide to maximal lipid-lowering therapy produced a significant reduction in LDL-C from baseline to Week 26 that was maintained throughout the study, and was generally well tolerated in the presence of dose titration and a low-fat diet. Gastrointestinal adverse events, which the most common treatment-emergent adverse effects, became less frequent and severe over time, and were managed through adherence to a low fat $(<20 \%)$ diet. Elevations in ALT and AST were managed with dose reduction and did not lead to any permanent treatment discontinuations. Hepatic fat content was increased at Week 26 as compared with baseline, but appeared to stabilise thereafter. Lomitapide enabled some patients to achieve target LDL-C levels and reduce the frequency of or stop apheresis treatment whilst maintaining LDL-C levels.

\section{Ongoing pharmacovigilance as clinical experience continues}

A Risk Management Plan has been developed for lomitapide in the US, Europe and Canada that includes 

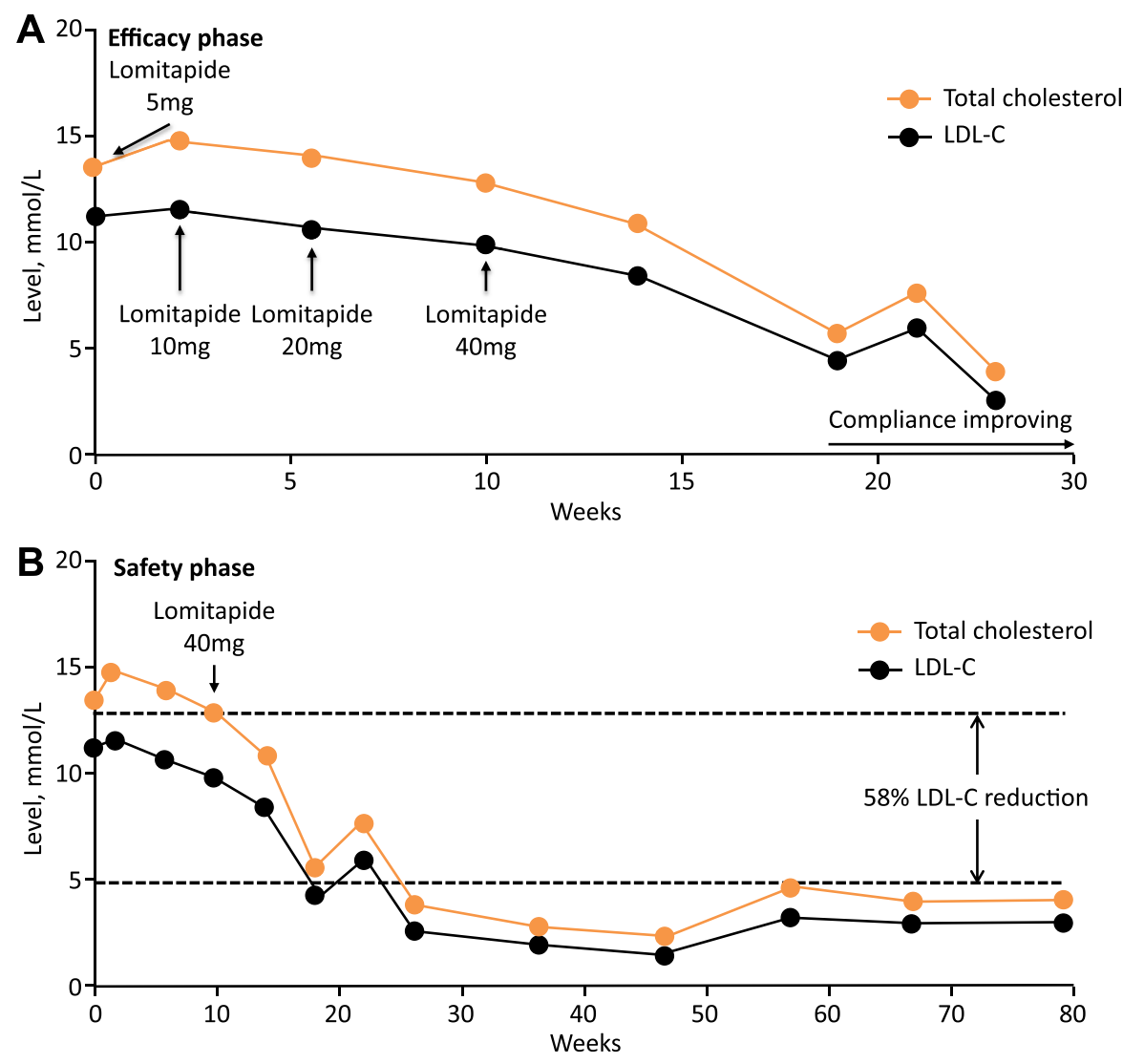

Fig. 6. Case study 2. Low-density lipoprotein cholesterol (LDL-C) and total cholesterol levels in a patient receiving lomitapide in phase 3 study. From lomitapide efficacy phase (A), through to extension phase (B).

educational materials for prescribers and patients. The Lomitapide Observational Worldwide Evaluation Registry (LOWER) is a global registry study that systematically collects information on the safety and effectiveness outcomes of all patients treated with lomitapide. Initiated in March 2014, it is anticipated that this study will enrol 300 patients and follow them for at least 10 years. The LOWER study has a number of objectives, including evaluation of adverse events occurring during long-term treatment with lomitapide and effectiveness in maintaining control of serum lipid levels in clinical practice. The occurrence and outcomes of pregnancy in female patients treated with lomitapide will also be evaluated in the pregnancy exposure registry (PER), a sub-registry of LOWER.

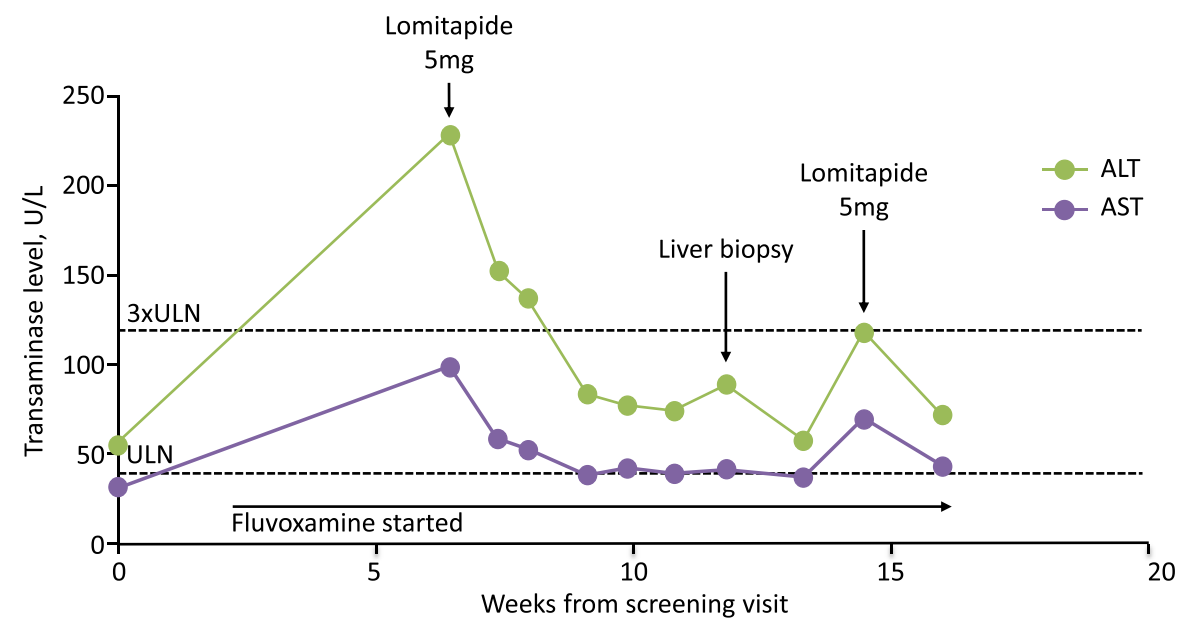

Fig. 7. Case study 3. Baseline levels of transaminases (ALT and AST) before treatment with lomitapide. The run-in period for this patient was longer than typical due to an AST/ALT flare associated with the first administration of lomitapide. The drug was stopped and successfully reintroduced 9 weeks later. 

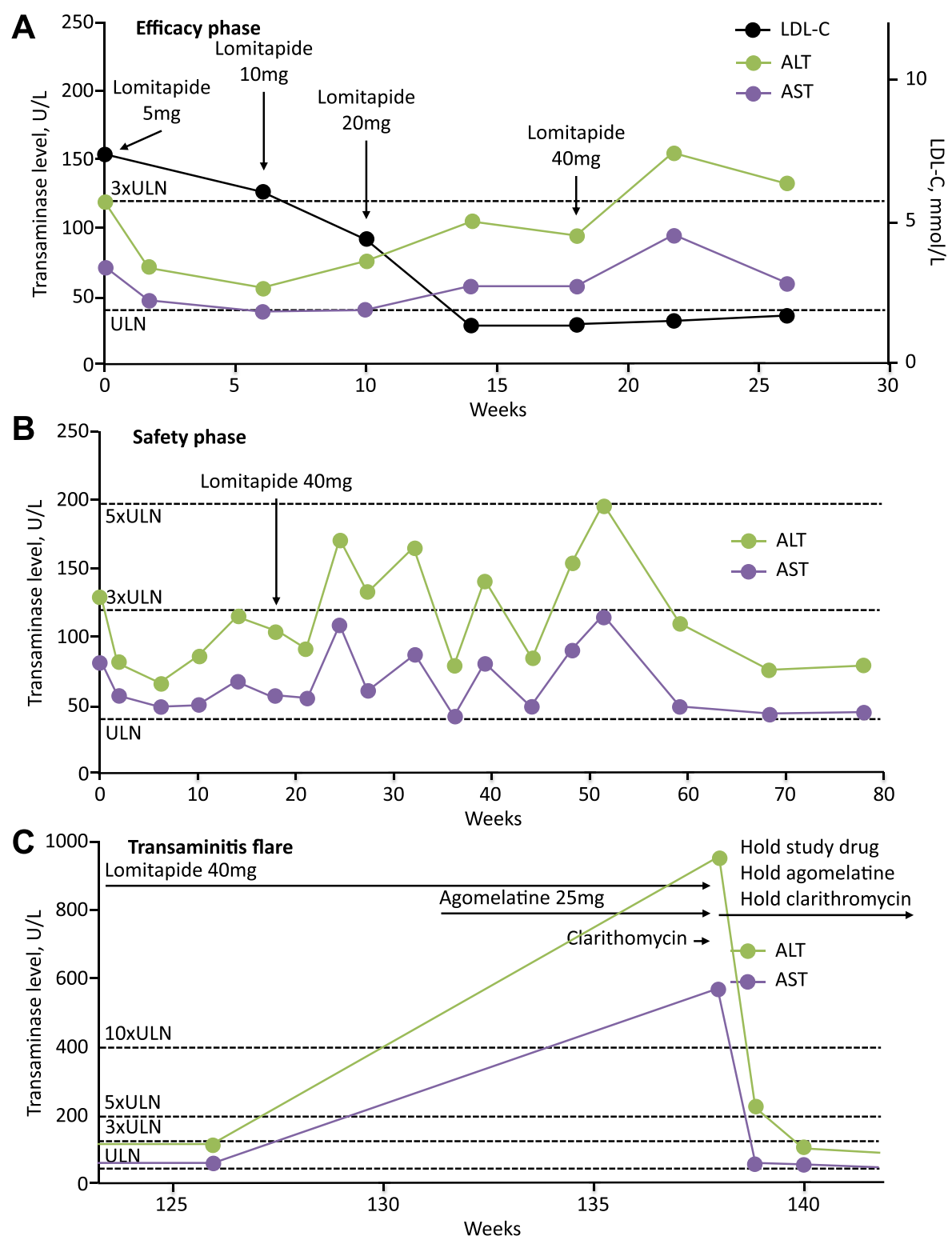

Fig. 8. Case study \#3: Concentrations of transaminases (ALT and AST) during efficacy phase (A), safety phase (B) and transaminitis flare (C).

\section{Questions from the audience during the educational symposium at EAS 2013}

Q: Lomitapide also inhibits the formation of chylomicrons. Does anyone have any experience of using lomitapide in patients with chylomicronaemia syndrome, and what is the effect in these patients?

A: At this time, we have no data on the effect of lomitapide in patients with chylomicronaemia syndrome. However, the patient who has received treatment with lomitapide for the longest time, under a compassionate use programme in the USA, is a woman with type 1 hyperlipidaemia (familial chylomicronaemia syndrome) that has caused severe recurrent pancreatitis. The patient has been receiving lomitapide since early in 2000 and this aspect of the symptomatology has improved quite dramatically.

Q: What is the impact of lomitapide on $\operatorname{Lp}(a)$ ?

A: We observed around $20 \%$ reduction of $L p(a)$ with lomitapide at Week 26 of the study. However the values returned to baseline by 78 weeks of therapy. The reasons for this are still not yet understood.

Q: In the Netherlands we have a database of $50 \mathrm{HoFH}$ patients who are genetically homozygous but less than half of the patients have baseline $L D L-C$ above $13 \mathrm{mmol} / \mathrm{L}$. How should we define these patients?

A: Historically, before genetic diagnosis was possible, patients were defined based on lipid levels and patients such as these may have been considered as patients with 'severe' heterozygous HoFH. However, based on genetic 


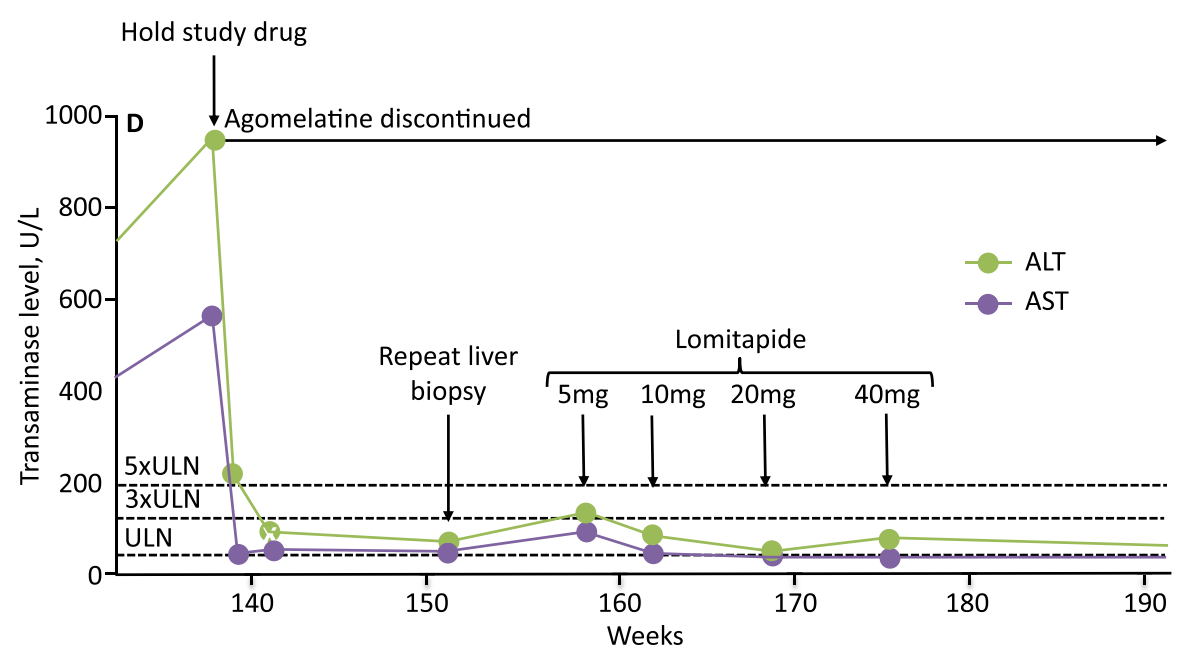

Fig. 9. Case study 3: Concentrations of transaminases (ALT and AST) during rechallenge with lomitapide.

profiling, these are clearly $\mathrm{HoFH}$ patients. $\mathrm{HoFH}$ is an extremely heterogeneous condition, partly explained by the large number $(>1600)$ and variety of LDL-receptor mutations identified thus far and there are likely yet undiscovered mutations in the LDL-receptor. Mutations of both alleles of the ARH gene can also cause HoFH and patients with ARH are often more responsive to statins than patients in whom HoFH is due to mutations in the LDLreceptor. Many other genes may also modify the phenotype and it is thus not surprising that there is a spectrum of severity that partially overlaps with heterozygous $\mathrm{FH}$.

\section{Questions from audience during the meet the expert session at EAS 2013}

Q: What proportion of dietary fat should we be aiming for?

A: When we counsel patients we should aim for $20 \%$ of calories from fat. In the clinical trial the mean fat intake achieved was $24 \%$.

Q: Is there a need for supplementation of lipid-soluble vitamins and omega-3 fatty acids?

A: Yes, supplementation of $400 \mathrm{IU}$ of vitamin $\mathrm{E}$ and approximately $200 \mathrm{mg}$ linoleic acid, $210 \mathrm{mg}$ alpha-

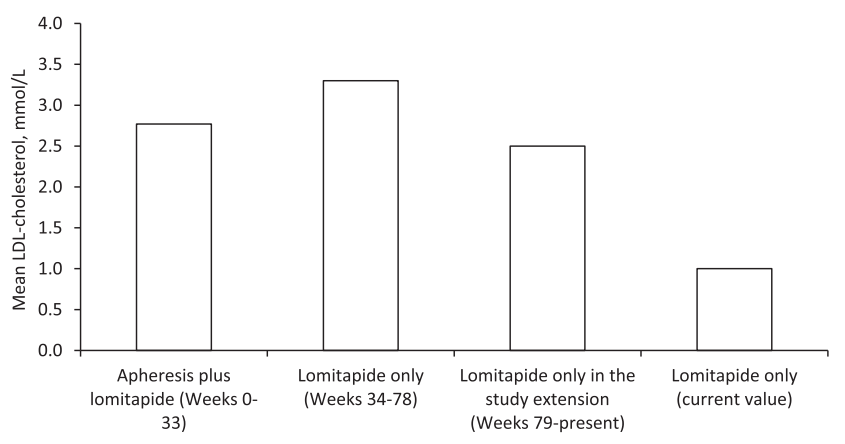

Fig. 10. Case study 4. Mean low-density lipoprotein (LDL) cholesterol levels in a patient receiving treatment with lomitapide, with or without LDL apheresis, in the phase 3 study. linolenic acid (ALA), $110 \mathrm{mg}$ eicosapentaenoic acid (EPA), and $80 \mathrm{mg}$ docosahexaenoic acid (DHA).

Q: In everyday clinical practice we do not have MRI facilities available. How do you monitor the liver in patients while they are taking lomitapide? A: The approved prescribing information for lomitapide advises that ALT, AST, alkaline phosphatase, and total bilirubin should be measured before initiating treatment and then ALT and AST regularly as recommended [15]. During treatment, if the ALT or AST are $\geq 3 \times$ ULN the dose of lomitapide should be adjusted according to the recommended algorithm. Lomitapide treatment should be discontinued if there is clinically significant liver toxicity. For hepatic fat, the EMA have recommended that this is monitored annually using imaging techniques such as fibroscan, acoustic radiation impulse (ARFI), or magnetic resonance (MR) elastography. This should be performed in addition to other tests including gamma-GT and serum albumin to detect possible liver injury and at least one marker from each of the following categories: High sensitivity C-reactive protein (hs-CRP), erythrocyte sedimentation rate (ESR), CK-18 Fragment, NashTest (liver inflammation) and one of the following: Enhanced Liver Fibrosis (ELF) panel, Fibrometer, AST/ALT ratio, Fib-4 score, Fibrotest (liver fibrosis). The performance of these tests and their interpretation should involve collaboration between the treating physician and the hepatologist. Patients with results suggesting the presence of steatohepatitis or fibrosis should be considered for liver biopsy.

Q: Can patients drink alcoholic beverages while taking lomitapide?

A: It is my view that they should avoid alcohol completely. The approved prescribing information in the US recommends that patients should consume no more than one alcoholic drink per day during treatment with lomitapide [15] however the European prescribing information states that due to the possible increase in hepatic fat and liver injury, alcohol is not recommended. [13] 
Q: What is the association between the increase in LFTs and accumulation of liver fat?

A: From the phase 3 study data it appears that increased ALT levels may be a marker for increased liver fat content. Hepatologists begin to be concerned when liver fat content is around $25-30 \%$ and levels seen in the phase 3 study were much lower than that. However, it is essential to be cautious and continue to monitor the patients in the long term.

A: There may need to be another trigger for progression from accumulation of bland fat to inflammation. Three of the four patients in the phase 3 trial who had marked LFT elevations were consuming excess alcohol. It is important to look out for other factors such as excess alcohol, excess iron and viral hepatitis, as they may trigger inflammation.

Q: Do the xanthomas regress with lomitapide treatment? This is something that patients like to see happening because of the impact on their appearance.

A: The cutaneous xanthomata definitely can regress and sometimes they disappear almost completely, as illustrated by the second case study in this paper. In our clinical experience we have not seen complete regression of tendinous xanthomata and it is hard to say objectively whether or not they have decreased in size. Clinical trials have not been designed to rigorously study xanthomata regression as an endpoint.

Q: Do we have any experience of lomitapide in paediatric patients?

A: No, we have no experience in paediatric patients. These data are needed.

Q: Do we have any experience of the use of lomitapide in pregnancy?

A: No, we have no experience of the use of lomitapide in pregnancy. At present lomitapide is contraindicated in patients who are pregnant.

Q: Is there any evidence from imaging studies to show regression of plaques with lomitapide?

A: The phase 3 study of lomitapide did not include an imaging component. However, patients enrolled in the LOWER registry study can participate in an openlabel study to assess changes in carotid and aortic atherosclerosis as assessed by magnetic resonance imaging.

\section{Conflict of interest}

M Averna: Maurizio Averna has served as a clinical investigator in several hypolipidemic drug trials for Aegerion, and is a member of Aegerion Europe and Italy Advisory Boards.

Marina Cuchel: Marina Cuchel has received reimbursement for the conduction of clinical trials, speaker honoraria and travel support for attending scientific meetings from Aegerion Pharmaceuticals.

\section{References}

[1] Catapano AL, Reiner Z, De Backer G, Graham I, Taskinen MR, Wiklund O, et al. European Society of Cardiology and European Atherosclerosis Society. ESC/EAS guidelines for the management of dyslipidaemias the task force for the management of dyslipidaemias of the European Society of Cardiology (ESC) and the European Atherosclerosis Society (EAS). Atherosclerosis 2011; 217:3-46.

[2] Raal FJ, Pilcher GJ, Panz VR, van Deventer HE, Brice BC, Blom DJ, et al. Reduction in mortality in subjects with homozygous familial hypercholesterolemia associated with advances in lipid-lowering therapy. Circulation 2011;124:2202-7.

[3] Bertolini S, Pisciotta L, Rabacchi C, Cefalu AB, Noto D, Fasano T, et al. Spectrum of mutations and phenotypic expression in patients with autosomal dominant hypercholesterolemia identified in Italy. Atherosclerosis 2013;227:342-8.

[4] Marais AD, Blom DJ, Firth JC. Statins in homozygous familial hypercholesterolemia. Current Atherosclerosis Reports 2002;4: $19-25$.

[5] Stein EA, Honarpour N, Wasserman SM, Xu F, Scott R, Raal FJ Effect of the proprotein convertase subtilisin/kexin 9 monoclonal antibody, AMG 145, in homozygous familial hypercholesterolemia. Circulation 2013;128:2113-20.

[6] Raal FJ, Santos RD, Blom DJ, Marais AD, Charng MJ, Cromwell WC, et al. Mipomersen, an apolipoprotein B synthesis inhibitor, for lowering of LDL cholesterol concentrations in patients with homozygous familial hypercholesterolaemia: a randomised, double-blind, placebo-controlled trial. Lancet 2010;375:998-1006.

[7] Cuchel M, Meagher EA, du Toit Theron H, Blom DJ, Marais AD, Hegele RA, et al. Phase 3 Ho FHLSi. Efficacy and safety of a microsomal triglyceride transfer protein inhibitor in patients with homozygous familial hypercholesterolaemia: a single-arm, openlabel, phase 3 study. Lancet 2013;381:40-6.

[8] Cuchel M, Meagher EA, du Toit Theron H, Blom DJ, Marais AD, Hegele RA, et al. Apheresis treatment does not affect the lipidlowering efficacy of lomitapide, a microsomal triglyceride transfer protein inhibitor, in patients with homozygous familial hypercholesterolemia. Circulation 2012;126:A17396.

[9] Gagné C, Gaudet D, Bruckert EEzetimibe Study G. Efficacy and safety of ezetimibe coadministered with atorvastatin or simvastatin in patients with homozygous familial hypercholesterolemia. Circulation 2002; 105:2469-75.

[10] Graesdal A, Bogsrud MP, Holven KB, Nenseter MS, Narverud I, Langslet G, et al. Apheresis in homozygous familial hypercholesterolemia: the results of a follow-up of all Norwegian patients with homozygous familial hypercholesterolemia. J Clin Lipidol 2012;6: $331-9$.

[11] Nordestgaard BG, Chapman MJ, Humphries SE, Ginsberg HN, Masana L, Descamps OS, et al. for the European Atherosclerosis Society Consensus Panel. Familial hypercholesterolaemia is underdiagnosed and undertreated in the general population: guidance for clinicians to prevent coronary heart disease: Consensus Statement of the European Atherosclerosis Society. Eur Heart J 2013;34: 3478-3490a.

[12] Bruckert E, Saheb S, Bonté JR, Coudray-Omnés C. Daily life, experience and needs of persons suffering from homozygous familial hypercholesterolaemia: Insights from a patient survey. Atherosclerosis Supplements 2014;15:46-51.

[13] Aegerion Pharmaceuticals Inc. Lojuxta summary of product characteristics; 2013.

[14] Tuteja S, Duffy D, Dunbar RL, Movva R, Gadi R, Bloedon LT, et al. Pharmacokinetic interactions of the microsomal triglyceride transfer protein inhibitor, lomitapide, with drugs commonly used in the management of hypercholesterolemia. Pharmacotherapy 2014;34: $227-39$. 
[15] Aegerion Pharmaceuticals Inc. Juxtapid prescribing information; 2013.

[16] Thompson GR, Barbir M, Davies D, Dobral P, Gesinde M, Livingston M, et al. Efficacy criteria and cholesterol targets for LDL apheresis. Atherosclerosis 2010;208:317-21.
[17] Kroon AA, van't Hof MA, Demacker PN, Stalenhoef AF. The rebound of lipoproteins after LDL-apheresis. Kinetics and estimation of mean lipoprotein levels. Atherosclerosis 2000;152: $519-26$. 